\title{
FURTHER NON-INVOLUTORIAL CREMONA SPACE TRANSFORMATIONS CONTAINED IN A SPECIAL LINEAR COMPLEX*
}

\section{BY AMOS BLACK}

1. Introduction. In a series of papers by Snyder, $\uparrow$ Carroll, $\ddagger, \S$ and the author, $\|, \mathbb{\Phi}$ involutorial transformations were defined by means of a correspondence between the surfaces of a pencil and the points of a rational curve. The purpose of this paper is to apply similar methods to certain non-involutorial transformations.

2. Definition of the Transformation. Given a line $d$ and two pencils of surfaces $\left|F_{n}\right|$ and $\left|F_{n^{\prime}}^{\prime}\right|$ of orders $n$ and $n^{\prime}$ which contain $d$ as an $(n-1)$-fold and $\left(n^{\prime}-1\right)$-fold line, respectively. Make the surfaces of each pencil projective with the points of $d$. A point $P$ will determine a unique surface $F_{n}$ passing through it, hence a unique point $O$ on $d$ and a unique surface $F_{n^{\prime}}^{\prime}$. The line $P O$ cuts $F_{n}^{\prime}$ in one point $P^{\prime}$ (other than $O$ ) which is defined as the image of $P$.

Since $P$ and $P^{\prime}$ lie on a line which intersects $d$, any plane through $d$ is transformed into itself. We shall find the plane transformation in an arbitrary plane through $d$ and then generate the space transformation by revolving the plane about $d$.

3. The Plane Transformation. The intersections of an arbi-

* Presented to the Society, December 27, 1933.

$\dagger$ Virgil Snyder, On a series of involutorial cremona transformations of space defined by a pencil of ruled surfaces, Transactions of this Society, vol. 35 (1933), pp. 341-347.

$\ddagger$ Evelyn Carroll, Systems of involutorial birational transformations contained multiply in special linear line complexes, American Journal of Mathematics, vol. 54 (1932), pp. 707-717.

$\S$ Evelyn Carroll-Rusk, Cremona involutions defined by a pencil of cubic surfaces, American Journal of Mathematics, vol. 56 (1934), pp. 96-108.

\| Amos Black, Types of involutorial space transformations associated with certain rational curves, Transactions of this Society, vol. 34 (1932), pp. 795810.

I Amos Black, Types of involutorial space transformations associated with certain rational curves-composite basis curves, this Bulletin, vol. 40 (1934), pp. 417-420. 
trary plane through $d$ with $\left|F_{n}\right|: d^{n-1}$ and $\left|F_{n^{\prime}}^{\prime}\right|: d^{n^{\prime}-1}$ are two pencils of lines $|l|: V$ and $\left|l^{\prime}\right|: V^{\prime}$, respectively, where $V$ and $V^{\prime}$ are the vertices of the pencils. The lines of each pencil are projective with the points of $d$. Thus a point $P$ determines a line $l$ through it, a point $O$ on $d$, and a line $l^{\prime}$. The point $P^{\prime}$, the image of $P$, is the intersection of $P O$ and $l^{\prime}$.

The directions through $V$ are perspective with the points of $d$ which are projective with the lines $\left|l^{\prime}\right|$. The conic $c_{2}^{\prime}$ of intersection of these two projective pencils is the image of $V$.

Since there is a $(1,1)$ correspondence between the lines of $|l|$ and the points of $d$, there are two coincidences, hence two lines $l_{3}, l_{4}$ which pass through their respective associated points $O_{1}$, $O_{2}$. Let us call the associated lines of $\left|l^{\prime}\right|, l_{1}^{\prime}$ and $l_{2}^{\prime}$, respectively. If we choose $P$ as an arbitrary point on $l_{3}$, its image is $P_{1}^{\prime}$, the point of intersection of $l_{3}$ and $l_{1}^{\prime}$. If we choose $P$ at $O_{1}$ the line $P O_{1}$ is indeterminate. However, we may readily find the image of $O_{1}$ from the inverse transformation. If we choose $P^{\prime}$ as an arbitrary point on $l_{1}^{\prime}$, then $P^{\prime} O_{1}$ intersects $l_{3}$ in $O_{1}$. Thus $O_{1}$ has for image the whole line $l_{1}^{\prime}$. Hence $l_{3}: V O_{1} P_{1}^{\prime} \sim c_{2}^{\prime} l_{1}^{\prime} P_{1}^{\prime}$. Similarly $l_{4}: V O_{2} P_{2}^{\prime} \sim c_{2}^{\prime} l_{2}^{\prime} P_{2}^{\prime}$. Beginning with $V^{\prime}$ and $\left|l^{\prime}\right|$, in a similar manner we find $l_{3}^{\prime}: V^{\prime} O_{1}{ }^{\prime} P_{1} \sim c_{2} l_{1} P_{1}$ and $l_{4}^{\prime}: V^{\prime} O_{2}^{\prime} P_{2}$ $\sim c_{2} l_{2} P_{2}$.

There are no other fundamental points or curves, hence the plane transformation is the well known $T_{3}: 1^{2} 4^{1}$.

$$
\begin{array}{lll}
T_{3}: V^{2} O_{1} O_{2} P_{1} P_{2}, & T_{3}^{\prime}: V^{\prime 2} O_{1}^{\prime} O_{2}^{\prime} P_{1}^{\prime} P_{2}^{\prime}, \\
J_{6}=c_{2} l_{1} l_{2} l_{3} l_{4}, & J_{6}^{\prime}=c_{2}^{\prime} l_{1}^{\prime} l_{2}^{\prime} l_{3}^{\prime} l_{4}^{\prime}, \\
V \sim c_{2}^{\prime}: V V^{\prime} O_{1}^{\prime} O_{2}^{\prime} P_{1}^{\prime} P_{2}^{\prime}, & V^{\prime} \sim c_{2}: V V^{\prime} O_{1} O_{2} P_{1} P_{2}, \\
O_{1} \sim l_{1}^{\prime}: V^{\prime} P_{1}^{\prime}, & O_{1}^{\prime} \sim l_{1}: V P_{1}, \\
O_{2} \sim l_{2}^{\prime}: V^{\prime} P_{2}^{\prime}, & O_{2}^{\prime} \sim l_{2}: V P_{2}, \\
P_{1} \sim l_{3}^{\prime}: V^{\prime} O_{1}^{\prime} P_{1}, & P_{1}^{\prime} \sim l_{3}: V O_{1} P_{1}^{\prime}, \\
P_{2} \sim l_{4}^{\prime}: V^{\prime} O_{2}^{\prime} P_{2}, & P_{2}^{\prime} \sim l_{4}: V O_{2} P_{2}^{\prime} .
\end{array}
$$

The invariant curve is the conic $k_{2}$, the intersection of the two projective pencils $|l|,\left|l^{\prime}\right|$. Hence $k_{2}: V V^{\prime} P_{1} P_{2} P_{1}^{\prime} P_{2}^{\prime}$.

4. The Space Transformation. Since the space transformation is generated by the plane $T_{3}$, except for orders, we can immediately determine the surfaces of the space transformation. As 
we revolve the plane of $T_{3}$ about $d$, the points $O_{1}, O_{2}$ generate $d$. Hence $l_{1}^{\prime}, l_{2}^{\prime}$ generate a surface $L^{\prime}$ which is the image of $d$. The points $P_{1}, P_{2}$ generate a curve $\delta$, whose image $X^{\prime}$ is the locus of $l_{3}^{\prime}, l_{4}^{\prime}$. The vertex $V$ generates the residual basis curve $\gamma$ of the pencil $\left|F_{n}\right|$, and the image $\Gamma^{\prime}$ of $\gamma$ is the locus of $c_{2}^{\prime}$. In a similar manner $\delta^{\prime}, L, X, \Gamma$ are obtained. The locus of $k_{2}$ is the invariant surface $K$.

Since the sections of all the surfaces of the space transformation with a plane through $d$ are known, except for the multiplicity of $d$, whatever the values of $n$ and $n^{\prime}$ may be, there is no advantage in having $n$ and $n^{\prime}$ large. We shall set up the analytic work for $n=n^{\prime}=2$.

5. Equations of the Transformation. Let the equations of $d$ be $x_{1}=0, x_{2}=0$, and let the coordinates of a point on $d$ be given by $O(0,0, \lambda, 1)$. Also let

$$
\begin{gathered}
|H(x)|=H_{1}(x)-\lambda H_{2}(x)=0 \\
\left|H^{\prime}(x)\right|=H_{1}^{\prime}(x)-\lambda H_{2}^{\prime}(x)=0
\end{gathered}
$$

where

$$
\begin{aligned}
H_{1}(x) & =x_{1} u_{1}(x)+x_{2} u_{2}(x), & H_{2}(x) & =x_{1} v_{1}(x)+x_{2} v_{2}(x), \\
H_{1}^{\prime}(x) & =x_{1} u_{1}^{\prime}(x)+x_{2} u_{2}^{\prime}(x), & H_{2}^{\prime}(x) & =x_{1} v_{1}^{\prime}(x)+x_{2} v_{2}^{\prime}(x), \\
u_{i}(x) & =\sum a_{i j} x_{j}, & v_{i}(x) & =\sum b_{i j} x_{j}, \\
u_{i}^{\prime}(x) & =\sum a_{i j}^{\prime} x_{j}, & v_{i}^{\prime}(x) & =\sum b_{i j}^{\prime} x_{j},
\end{aligned}
$$

for $i=1,2$ and $j=1,2,3,4$. Since the parameters of the points of $d$ and the surfaces of (1) and (2) are identical, the forms are projective.

The surface of (1) passing through $P(x)$ gives $\lambda=H_{1} / H_{2}$, where $H_{1}=H_{1}(x)$ and $H_{2}=H_{2}(x)$, and the associated point on $d$ is $O(z)=\left(0,0, H_{1}, H_{2}\right)$, and the associated surface of $(2)$ is

$$
H_{2} H_{1}^{\prime}(x)-H_{1} H_{2}^{\prime}(x)=0 .
$$

The coordinates of any point on the line $P O$ are given by

$$
x_{i}^{\prime}=\rho x_{i}+z_{i}, \quad(i=1,2,3,4) .
$$

Substituting from (4) in (3) gives $\rho=L_{5} / K_{4}$, where 


$$
\begin{aligned}
L_{5}= & H_{2}^{2}\left(a_{14}^{\prime} x_{1}+a_{24}^{\prime} x_{2}\right)+H_{1} H_{2}\left(a_{13}^{\prime} x_{1}+a_{23}^{\prime} x_{2}\right. \\
& \left.-b_{14}^{\prime} x_{1}-b_{24}^{\prime} x_{2}\right)-H_{1}^{2}\left(b_{13}^{\prime} x_{1}+b_{23}^{\prime} x_{2}\right), \\
K_{4}= & H_{2} H_{1}^{\prime}-H_{1} H_{2}^{\prime} .
\end{aligned}
$$

The equations of the transformation are

$$
T_{6}: \quad x_{i}^{\prime}=x_{i} L_{5}-K_{4} z_{i}, \quad(i=1,2,3,4) .
$$

It is evident from the form of the equations of $T_{6}$ that $K_{4}=0$ is the surface of invariant points, and that $L_{5}=0$ is the image of $d$.

Any arbitrary plane $\pi$ through $d$ is the tangent plane of two surfaces of $\left|H^{\prime}(x)\right|$ at points on $d$. The two points of contact are the points $O_{1}^{\prime}, O_{2}^{\prime}$ of the plane $T_{3}$ in $\pi$. The intersections of $\pi$ with the two surfaces of $\left|H^{\prime}(x)\right|$ associated with $O_{1}^{\prime}, O_{2}^{\prime}$ are the lines $l_{3}^{\prime}, l_{4}^{\prime}$ and the intersections with the associated surfaces of $|H(x)|$ are the lines $l_{1}, l_{2}$. Since $L_{5}$ is the locus of $l_{1}, l_{2}$, then the elimination of $\lambda$ between the equations of the tangent plane of a surface $H^{\prime}(x)$ at its associated point $O$ and the associated surface $H(x)$ gives $L_{5}$.

The tangent plane of $H^{\prime}(x)$ at its associated point $O$ is

$$
\begin{aligned}
& {\left[a_{13}^{\prime} \lambda+a_{14}^{\prime}-\lambda\left(b_{13}^{\prime} \lambda+b_{14}^{\prime}\right)\right] x_{1}} \\
& \quad+\left[a_{23}^{\prime} \lambda+a_{24}^{\prime}-\lambda\left(b_{23}^{\prime} \lambda+b_{24}^{\prime}\right)\right] x_{2}=0 .
\end{aligned}
$$

The elimination of $\lambda$ between (7) and (1) gives $L_{5}$. Similarly, since the locus of $l_{3}^{\prime}, l_{4}^{\prime}$ is $X_{5}^{\prime}$, then the elimination of $\lambda$ between (7) and (2) gives $X_{5}^{\prime}$, or

$$
\begin{aligned}
X_{5}^{\prime}= & H_{2}^{\prime 2}\left(a_{14}^{\prime} x_{1}+a_{24}^{\prime} x_{2}\right)+H_{1}^{\prime} H_{2}^{\prime}\left(a_{13}^{\prime} x_{1}+a_{23}^{\prime} x_{2}\right. \\
& \left.-b_{14}^{\prime} x_{1}-b_{24}^{\prime} x_{2}\right)-H_{1}^{\prime 2}\left(b_{13}^{\prime} x_{1}+b_{23}^{\prime} x_{2}\right) .
\end{aligned}
$$

Since any quadric of $\left|H^{\prime}(x)\right|$ is transformed into the associated quadric of $|H(x)|$, the equation of the image of $\gamma_{3}^{\prime}$ may be found by transforming $H_{1}^{\prime}(x)$. Hence $H_{1}^{\prime}(x) \sim H_{1} L_{5} \Gamma_{5}$, where

$$
\begin{aligned}
\Gamma_{5}= & {\left[H_{2}^{\prime}\left(a_{14}^{\prime} x_{1}+a_{24}^{\prime} x_{2}\right)-H_{1}^{\prime}\left(b_{14}^{\prime} x_{1}+b_{24}^{\prime} x_{2}\right)\right] H_{2} } \\
& +\left[H_{2}^{\prime}\left(a_{13}^{\prime} x_{1}+a_{23}^{\prime} x_{2}\right)-H_{1}^{\prime}\left(b_{13}^{\prime} x_{1}+b_{23}^{\prime} x_{2}\right)\right] H_{1} .
\end{aligned}
$$

In a similar manner we may write the equations of

$$
T_{6}^{\prime}: \quad x_{i}=x_{i}^{\prime} L_{5}^{\prime}+K_{4} z_{i}^{\prime}, \quad(i=1,2,3,4),
$$

where $\left(z_{i}^{\prime}\right)=\left(0,0, H_{1}^{\prime}, H_{2}^{\prime}\right)$ and $L_{5}^{\prime}, X_{5}, \Gamma_{5}^{\prime}$ are found from $L_{5}$, $X_{5}^{\prime}, \Gamma_{5}$ by interchanging primes and unprimes. 
The multiplicates of $\gamma_{3}, \gamma_{3}^{\prime}, \delta_{8}, \delta_{8}^{\prime}$ on the various surfaces are known from $T_{3}$, and the multiplicity of $d$ can easily be verified from equations (5) to (10). Of the three tangent planes of any homoloid at any point on $d$, one tangent plane is the tangent plane of the associated quadric at the point. Thus the homoloidal surfaces have simple contact along $d$. Collecting these results, we have

$$
\begin{aligned}
\text { a plane } & \stackrel{T}{\sim} S_{6}: d^{3+1 t} \gamma_{3}{ }^{2} \delta_{8}, & \text { a plane } & \stackrel{T^{\prime}}{\sim} S_{6}^{\prime}: d^{3+1 t} \gamma_{3}^{\prime 2} \delta_{8}^{\prime}, \\
d & \sim L_{5}: d^{3} \gamma_{3}{ }^{2} \delta_{8}, & d & \sim L_{5}^{\prime}: d^{3} \gamma_{3}^{\prime}{ }^{2} \delta_{8}^{\prime}, \\
\gamma_{3}^{\prime} & \sim \Gamma_{5}: d^{3} \gamma_{3} \gamma_{3}^{\prime} \delta_{8}, & \gamma_{3} & \sim \Gamma_{5}^{\prime}: d^{3} \gamma_{3} \gamma_{3}^{\prime} \delta_{8}^{\prime}, \\
\delta_{8}^{\prime} & \sim X_{5}: d^{3} \gamma_{3}{ }^{2} \delta_{8}^{\prime}, & \delta_{8} & \sim X_{5}^{\prime}: d^{3} \gamma_{3}^{\prime 2} \delta_{8}, \\
& K_{4}: d^{2} \gamma_{3} \gamma_{3}^{\prime} \delta_{8} \delta_{8}^{\prime} . & &
\end{aligned}
$$

6. Special Cases. A pencil of quadrics whose base is a line and a twisted cubic contains two cones. In general, the vertex of the cone is not at its associated point in our projectivity. Whenever the vertex and the associated point coincide we have a special case.

CASE 1. Suppose $b_{13}^{\prime}=b_{23}^{\prime}=0$. Then $H_{2}^{\prime}(x)$ is a cone with vertex $O_{1}^{\prime}(0,0,1,0)$, and from (2), we see that the vertex and associated point coincide. In the pencil $\left|l^{\prime}\right|$ of the plane $T_{3}$, one line is a generator of the cone, hence passes through its associated point $O_{1}^{\prime}$. The image of $O_{1}^{\prime}$ is the line $l_{1}$ of $H_{2}$ lying in this plane. The image of the generator is $P_{1}$, the point of intersection of the generator and $l_{1}$. But as the plane is rotated about $d$, the point $O_{1}^{\prime}$ remains fixed, $l_{1}$ generates $H_{2}$, and $P_{1}$ generates a twisted cubic $\delta_{3}$. Thus, in space, $O_{1}^{\prime} \sim H_{2}$, and $H_{2}^{\prime} \sim \delta_{3}$. However, the other incidence point $O_{2}^{\prime}$ varies and generates $d$, and $P_{2}$ varies and generates a rational curve $\delta_{5}$. It is easily seen from (5) that if $b_{13}^{\prime}=b_{23}^{\prime}=0$, then $L_{5}=H_{2} L_{3}$, and from (8) that $X_{6}^{\prime}=H_{2}^{\prime} X_{3}^{\prime}$. Then, in this special sense, in $T_{6}^{\prime}$ an isolated fundamental point $O_{1}^{\prime}$ with image $H_{2}$ is added, and in $T_{6}$ the fundamental curve $\delta_{8}$ is composite with $\delta_{3} \sim H_{2}^{\prime}$ and $\delta_{5} \sim X_{3}^{\prime}$.

CASE 2. If a cone of $\left|H^{\prime}(x)\right|$ has its vertex at its associated point and also a cone of $|H(x)|$ has its vertex at its associated point, but the vertices are distinct, then we have the specialization of Case 1 in both transformations. That is, both $T_{6}$ and $T_{6}^{\prime}$ have an isolated fundamental point on $d$, and both $\delta_{8}$ and $\delta_{8}^{\prime}$ are composite. 
CAsE 3. Let $|H(x)|$ and $\left|H^{\prime}(x)\right|$ both have a cone with vertex at its associated point, and let the vertices coincide. Let the associated point be $O(0,0,1,0)$ and let the cones be $H_{2}$ and $H_{2}^{\prime}$. Then $b_{13}=b_{23}=b_{13}^{\prime}=b_{23}^{\prime}=0$. In the plane $T_{3}$ this means that $O_{1}, O_{1}^{\prime}, P_{1}, P_{1}^{\prime}$ all coincide with $O$ and that $l_{1}=l_{3}, l_{1}^{\prime}=l_{3}^{\prime}$. In space $L_{5}=H_{2} L_{3}, X_{5}=H_{2} X_{3}, L_{5}^{\prime}=H_{2}^{\prime} L_{3}^{\prime}, X_{5}^{\prime}=H_{2}^{\prime} X_{3}^{\prime}$.

Since $\mathrm{O}_{2}, \mathrm{O}_{2}^{\prime}, \mathrm{P}_{2}, \mathrm{P}_{2}^{\prime}, \mathrm{V}, \mathrm{V}^{\prime}$ are variable, then as the plane of $T_{3}$ is revolved about $d$, these points generate $d, d^{\prime}, \delta_{5}, \delta_{5}^{\prime}, \gamma_{3}$, $\gamma_{3}^{\prime}$, respectively, and their respective images are $L_{3}^{\prime}, L_{3}, X_{3}^{\prime}$, $X_{3}, \Gamma_{5}^{\prime}, \Gamma_{5}$. The point $O$ is fixed and $O \stackrel{T^{T}}{\sim} H_{2}^{\prime}$ and $O \stackrel{T^{\prime}}{\sim} H_{2}$. The two cones $H_{2}$ and $H_{2}^{\prime}$ intersect in $d$ and in three other lines $l_{i}$, $(i=1,2,3)$. Each of these lines is parasitic; hence $\delta_{3}=\delta_{3}^{\prime}=3 l$.

CAse 4. Let $\left|H^{\prime}(x)\right|$ have two cones with their vertices at their associated points. Let the two cones be $H_{2}^{\prime}$ with vertex $O_{1}^{\prime}(0,0,1,0)$ and $H_{1}^{\prime}$ with vertex $O_{2}^{\prime}(0,0,0,1)$. Then we have $b_{13}^{\prime}=b^{\prime}{ }_{3}=a_{14}^{\prime}=a_{24}^{\prime}=0$. We find $L_{5}=H_{1} H_{2} \pi, X_{5}^{\prime}=H_{1}^{\prime} H_{2}^{\prime} \pi$, where $\pi=a_{13}^{\prime} x_{1}+a_{23}^{\prime} x_{2}-b_{14}^{\prime} x_{1}-b_{24}^{\prime} x_{2}$. Then the equations of $T_{6}$ may be written in the form

$$
x_{i}^{\prime}=x_{i} H_{1} H_{2} \pi-K_{4} z_{i}, \quad(i=1,2,3,4) .
$$

As we would expect from Case 1, $\mathrm{O}_{1}^{\prime} \sim H_{2}, \mathrm{O}_{2}^{\prime} \sim H_{1}, H_{2}^{\prime} \sim \delta_{3}$, $H_{1}^{\prime} \sim \bar{\delta}_{3}$.

As we rotate the plane $T_{3}$ about $d$ the points $O_{1}^{\prime}, O_{2}^{\prime}$ remain fixed, so apparently no other points of $d$ are fundamental, and hence $d$ has no image; yet from the form of equations (11) the plane $\pi$ is the image of $d$. The plane $\pi$ is the tangent plane of every surface of $\left|H^{\prime}(x)\right|$ at its associated point. Hence in the plane $T_{3}$ in plane $\pi$, every line of $\left|l^{\prime}\right|$ passes through its associated point. Therefore, in plane $\pi, d \stackrel{T^{\prime}}{\sim} \pi$ and $\pi \stackrel{T}{\sim} \delta_{2}$, where $\delta_{2}$ is the conic of intersection of the two projective pencils in $\pi$.

Then in $T_{6}^{\prime}$ we introduce two isolated fundamental points, $O_{1}^{\prime}$ with image $H_{2}$ and $O_{2}^{\prime}$ with image $H_{1}$, and $d \sim \pi$; while in $T_{6}$ the curve $\delta_{8}$ becomes composite, consisting of $\delta_{3}, \bar{\delta}_{3}, \delta_{2}$ with images $H_{2}^{\prime}, H_{1}^{\prime}, \pi$, respectively.

We may have combinations of Cases 1, 3, 4 just as Case 2 was a combination of a Case 1 with a Case 1 .

7. Special Cases due to Composite Basis Curves. The only way in which $\gamma_{3}$ and $\gamma_{3}^{\prime}$ can be composite is that the composite curve consist of a line, or lines, meeting $d$ and a residual curve. 
CASE 5. Let $\gamma_{3}=\gamma_{1} \gamma_{2}$, where $\gamma_{1}: x_{1}=0, x_{3}=0$ and $\left[\gamma_{1}, d\right]$

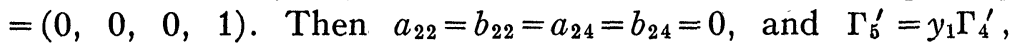
$X_{5}=y_{1} X_{4}$. No other surfaces of either $T_{6}$ or $T_{6}^{\prime}$ are composite.

Let us consider the plane $T_{3}$ in the plane $y_{1}=0$. The lines $\gamma_{1}, d$ form the complete intersection of any $H$ of $|H(x)|$ with the plane. Call the associated point of the composite $H$, of which $y_{1}$ is a component, $\bar{O}$. Fix a point $P$ on $\gamma_{1}$. The line $P \bar{O}$ meets $l_{\bar{O}}{ }^{\prime}$ in a point $P^{\prime}$, the image of $P$. As $\bar{O}$ traces $d, P^{\prime}$ generates a conic $c_{2}: P V^{\prime} \bar{O}_{1}^{\prime} \bar{O}_{2}^{\prime}$. As $P$ traces $\gamma_{1}, c_{2}$ generates the plane $y_{1}$. Hence $\gamma_{1} \sim$ the plane. Consider an arbitrary point $Q$ of the plane. The $H$ determined by $Q$ is composite and $\bar{O}$ is the associated point. The line $Q \bar{O}$ meets $l_{\bar{O}}{ }^{\prime}$ in $Q^{\prime}$, the image of $Q$. Then the whole line $Q \bar{O}$ transforms into $Q^{\prime}$, and the whole plane transforms into $l_{\bar{o}^{\prime}}$. Thus the curve $\delta_{8}^{\prime}$ is composite and consists of $\delta_{1}^{\prime}=l_{0}^{\prime}$ and a $\delta_{7}^{\prime}$.

If $\gamma_{3}$ consists of a line $\gamma_{1}$ meeting $d$ and a conic $\gamma_{2}$, then necessarily $\delta_{8}^{\prime}$ consists of a $\delta_{7}^{\prime}$, and a line $\delta_{1}^{\prime}$ in the plane of $\gamma_{1}, d$; and the plane is a principal surface for both $T_{6}$ and $T_{6}^{\prime}$.

CASE 6. If $\gamma_{3}$ consists of two lines each meeting $d$, and a third line skew to $d$, then the two planes determined by the two lines and $d$ are principal for both $T_{6}$ and $T_{6}^{\prime}$, and $\delta_{8}^{\prime}$ consists of a $\delta_{6}^{\prime}$ and two lines, one in each of the principal planes.

CASE 7. If $\gamma_{3}=\gamma_{1} \gamma_{2}$ and $\gamma_{3}^{\prime}=\gamma_{1}^{\prime} \gamma_{2}^{\prime}$, but $\gamma_{1}$ and $\gamma_{1}^{\prime}$ lie in different planes, then each plane is principal in both $T_{6}$ and $T_{6}^{\prime}$, and $\delta_{8}^{\prime}=\delta_{7}^{\prime} \delta_{1}^{\prime}$ and $\delta_{8}=\delta_{7} \delta_{1}$.

CASE 8. If $\gamma_{3}=\gamma_{1} \gamma_{2}, \gamma_{3}^{\prime}=\gamma_{1}^{\prime} \gamma_{2}^{\prime}$ and $\gamma_{1}, \gamma_{1}^{\prime}$ lie in the same plane but meet $d$ in distinct points, then the result is the same as in Case 7 except that $\gamma_{1}=\delta_{1}$ and $\gamma_{1}^{\prime}=\delta_{1}^{\prime}$.

The restriction that $\gamma_{1}, \gamma_{1}^{\prime}$ meet $d$ in distinct points is not necessary. If they meet $d$ in the same point, or even coincide, the associated points $\bar{O}, \bar{O}^{\prime}$ of the composite quadrics are, in general, distinct, hence the results are the same.

There may be special cases due to combinations of cases of $\S 6$ with cases of $\S 7$, but nothing new is added.

The line $d$ may be replaced by a conic $r_{2}$ or a twisted cubic $r_{3}$ with the restriction that transformations exist for $n=n^{\prime}=2$ only. However, the transformations are of the same form as the above, and no new results are obtained.

Cornell University 